Original Research Paper

\title{
Bit Error Rate Analysis of RS $(7,3)$ Coded Frequency Shift Keying Using Simulink
}

\author{
Shanmugasundaram T.A. and V. Vijayabaskar \\ Department of Electronics and Telecommunication Engineering, Faculty of Electrical and Electronics, \\ Sathyabama University, Chennai-600119, India
}

\author{
Article history \\ Received: 03-12-2014 \\ Revised: 16-03-2015 \\ Accepted: 17-03-2015 \\ Corresponding Author: \\ T.A. Shanmugasundaram \\ Department of Electronics and \\ Telecommunication \\ Engineering, Faculty of \\ Electrical and Electronics, \\ Sathyabama University, \\ Chennai-600119, India \\ Email:shamjan@gmail.com
}

\begin{abstract}
Bit Error Rate is an essential parameter to quantize the effectiveness of transceiver circuits used in low power, low duty cycle sensor nodes. BER can figure out their performance in terms of number of retransmissions, which keeps the node to consume its battery power undesirably rather than conserving it. Forward Error Correction schemes ensure the transceiver circuit to effectively perform its operations over a noisy bandwidth like ISM and under harsh/unpredictable channel conditions. As none of the coherent M-ary modulation schemes are 100\% efficient, non-coherent schemes can be considered as power-efficient choice for power limited sensor nodes. BFSK helps the sensor no des to have quicker transition from sleep mode to active mode. Otherwise, this will also force the transceiver to waste considerable amount of power. In this paper, by developing a SIMULINK model for RS $(7,3)$ coded BFSK in its non-coherent detection mode is analyzed for its bit error rate performance under various channel and coding conditions. Simulation results show that, this model gives its best BER performance as $16.67 \%$ under suitable conditions.
\end{abstract}

Keywords: Bit Error Rate, Low Power, Low Duty Cycle, Forward Error Correction, BFSK, RS Code

\section{Introduction}

The well-known channel impairments in wireless communication-fading of signals and interference from adjacent channels make the communication link to become weak. In order to have successful data transmission and reception in such an environment, much improved reliability and security (Wang et al., 2010; Mahajan and Singh, 2011) are in desire need. So it is mandatory to have effective strategies that provide both security protection and reliability assurance on data reception through error correcting codes and/or control mechanisms such as Automatic Repeat-Re Quest (ARQ) or Forward Error Correcting (FEC) codes.

According to ARQ mechanism, sender needs to retransmit the frame/packet until the reception of an acknowledgement. This introduces additional overhead such as delay and complexity. The time-varying and error-prone nature of a wireless channel causes frame loss/error which (Vibha and Nag, 2013) degrades the performance of the network.

Bit Error Rate (BER) (Khan et al., 2011) is the parameter to measure performance of digital data transmission and storage operations. Signal-to-Noise Ratio (SNR) is closely associated with BER such that, less the BER is better the SNR. During its passage through channel, signal gets distorted due to channel impairments like noise, interference, fading etc. Because of this, the actual data bits get altered and impose a severe problem to accuracy and performance of the digital communication system. With the help of an effective channel coding to do error detection and correction, a robust communication system can be designed to reduce the effects of a noisy transmission channel (Korrapati et al., 2013).

Development of channel coding requires knowledge on Error control coding theory because for each physical channel, its capacity $\mathrm{C}$ is a parametric quantity and a function of input-output characteristics of the channel. In order to meet the two basic and essential objectives, error detection and correction at the receiver (Shrivastava and Singh, 2013), the number of symbols in the source encoded message should be increased in a controlled manner. Signal-to-noise ratio describes the level of error detection and correction. 
Channel coding is referred as Forward Error Correction. FEC facilitates the receiver to detect and correct errors without any additional data from the sender. For this, sufficient amount of redundant data is added by the sender itself. Wherever retransmissions are much costly or not possible, FEC is the best solution. Among the several block codes like Golay, BCH, multidimensional parity and Hamming codes, Reed Solomon coding is widely used.

Because of the nature of correcting burst errors in mass storage devices (hard disk drives, DVD, barcode tags), wireless and mobile communications units, satellite links, digital TV, Digital Video Broadcasting (DVB) and modem technologies like xDSL, it can be used for data transmission and reception in wireless sensor networks, because sensor nodes are transmitting their data in burst not a periodic transmission. As the senor nodes are turned into sleep mode for most of the time and it will be continuously monitoring the physical environment of interest. It is turned to active mode only when it is to transmit its sensory data. In order to achieve reliable data transmission in WSNs, innovative RS codebased approaches are to be proposed.

The long burst errors (Kumar and Gupta, 2011) due to fast fading, leads to multipath effect and reduces the performance of WSN data transmission. To enhance the resilience of wireless links to these kinds of channel impairments, RS FEC is widely used, due to its excellent burst error correction properties. By mitigating the random bit errors that tend to dominate at low receiver SNR and minimum input power, RS FEC is the best choice (Vibha and Nag, 2013). Improving link margin requires either increased transmitter output power or reduced receiver noise figure. As both of these require more transmitter or receiver power, which degrades the lifetime of battery in low power wireless sensor network, applying FEC technique can offer a better system tradeoff without undesirably increased power requirement.

Puncturing of Reed Solomon code is also considered here for performance comparison so as to have fair trade-off between power consumption and performance. Even though, puncturing makes the transceiver circuit to be more flexible, it slightly increases the hardware complexity. Puncturing also helps the decoder to have less redundancy irrespective the number of bits punctured.

Interleaving reduces the damage caused to the data sequence by dispersing the occurrence of errors. Even though interleaving does not impose any additional bandwidth overhead, decoding latency is considerably increased as the receiver has to wait till all the data packets get buffered before rearranging. After resequencing the encoded data before transmission, the adjacent data units are separated by a predetermined distance. By using the distance and the way by which the data units are re-sequenced, the original sequence can be recovered at the receiver.

A method that encrypts only the major portion of the data with the security level required for encrypting entire data is proposed in (Wang et al., 2010) which significantly reduces computational overhead/delay and energy cost. Vibha and Nag (2013), the link margin and communication range improvement capability of RS codes for low power wireless networks is evaluated and performance metrics for latency and processing time needed to do RS encoding and decoding are also evaluated. BPSK scheme over AWGN channel and the resultant BER and SNR are evaluated through simulations in (Khan et al., 2011). A RS code with an error correction capability of 32 bits is proposed in (Shrivastava and Singh, 2013) with the focus on IEEE 802.15.4 wireless sensor network. With the aim of reducing $82 \%$ of erroneous bits, this error correction code improves Packet Error Rate (PER) over a communication distance not more than $40 \mathrm{~m}$ and with a targeted PER of $10^{-3}$, this coding scheme increases either the communication distance by $10 \mathrm{~m}$ or the RSSI by $8 \mathrm{~dB}$. The impact of code length ( $n$ ) of RS code over BER performance of M-ary FSK over a noisy communication channel is observed through Monte-Carlo simulations in (Mahajan and Singh, 2011) and another aspect of improved BER performance by lowering code rate is also observed. Through code interleaving which enables the long burst errors to be represented either as single random error or a short burst error is considered for the observation of BER performance of interleaved RS code symbols in (El-Bendary et al., 2014). In this simulation, the channel is assumed to be a Jakes channel. A simulation comparison between RS codes with varying $(\mathrm{n}, \mathrm{k})$ values is carried out in (Nguyen et al., 2012) for image transmission over wireless sensor networks. The results conclude that RS codes with larger $(n, k)$ values performs better than that with less $(n, k)$ values like $(7,3)$, $(15,7)$. But increased $(n, k)$ values require a complex hardware for its implementation.

The rest of the paper is organized as follows: Section II describes the Simulink model used in this simulation. Section III gives the complete detail about the simulation parameters used. Section IV provides the complete analysis on the simulation results. Section V concludes this paper.

\section{Simulink Model}

The output from Random integer generator block is the replica of Digital form of data sensed by the sensor nodes over the physical environment of interest. As shown in Fig. 1, the output from Random Integer generator block is encoded by $\mathrm{RS}(7,3)$ encoder with binary input. The encoded data sequence is interleaved by the Convolutional Interleaver block. 


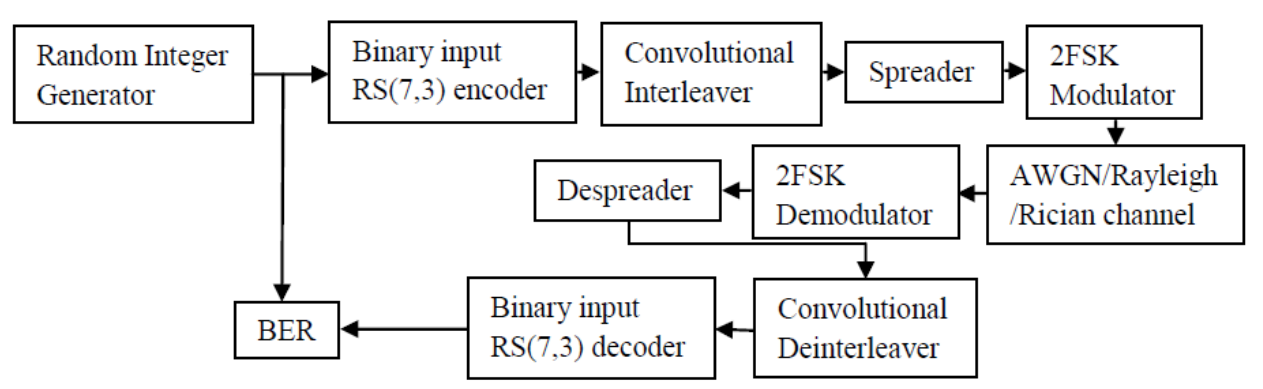

Fig. 1. Simulink block diagram

Direct Sequence Spread Spectrum technique is used to spread the spectrum of Interleaved cum encoded data. For this, the encoded cum interleaved data sequence is multiplied with a pseudo random noise sequence. The output from the spreader block modulates the carrier in 2ary FSK i.e., BFSK base band modulator. This modulated data is transmitted through the channel to the receiver.

At the receiver end, the received data is first demodulated by corresponding 2-ary FSK base band demodulator. After demodulation, the ensemble of data sequence is despreaded, deinterleaved before getting decoded by the corresponding Binary-input RS $(7,3)$ decoder. This decoded data sequence is compared with the originally transmitted data sequence for calculating the amount of erroneous bits received in terms of Bit Error Rate.

\section{Simulation Parameters}

Random Integer Generator generates its output for M $=2$ and Samples per frame $=9$, preferably number of bits per integer set in integer to bit converter block multiplied with the value of ' $\mathrm{k}$ ' set in RS encoder block.

The sample time is set suitably. In RS $(7,3)$ encoder block, the set primitive polynomial is:

$$
g(x)=x^{3}+x+1
$$

The default zeroes and ones are set for puncturing the RS $(7,3)$ code.

The convolutional interleaver used here is with 6 rows of shift register with a register length step of 2 . The PN sequence generator block is set with generator polynomial with mandatory coefficients and minimum initial states. The sample time is set in accordance to that set in Random Integer Generator block. The modulator block is set with $\mathrm{M}=2$ (the minimum value for less complex implementation), frequency separation between modulated components $=300 \mathrm{~Hz}$ with continuous phase and Samples per symbol $=12$.

For AWGN channel, Signal-to-noise ratio in terms of $\mathrm{E}_{\mathrm{b}} / \mathrm{N}_{\mathrm{o}}$ is varied over a suitable range for a particular value of number bits per symbol, which in turn is finalised by setting $E_{b} / N_{o}=0 d B$. Input signal power is set to any low value and the symbol period is set suitably. The parameters for the blocks at the receiving end are set in accordance with those set for the blocks at the transmitting end. Even though, the performance of FSK schemes is not affected by multipath Rayleigh and Rician fading and Doppler Shift, a minimum, non-zero value for the parameters relevant to these effects is used here.

For Rayleigh fading channel, initially average path gain vector is set to $\left[\begin{array}{ll}0 & 0\end{array}\right] \mathrm{dB}$ and average path delay vector is varied over a specific range to record the corresponding BER value. The value of average path gain vector is also varied over a specific range with approximately fixed average path delay vector as average path gain vector spreads over the average path delay vector. Any suitable non-zero value for Doppler Shift is set. The initial seed value is set in accordance with that set in Random Integer Generator.

For Rician fading channel, k-factor, the ratio between direct component and diffuse component is to be set suitably. Here it is set as 1 . Any suitable non zero value is set, preferably equal, for both Doppler Shift of LOS component and Maximum diffuse Doppler Shift with initial phase of LOS component $=0$. Bit Error Rate calculation is done with zero computation and receive delay.

\section{Simulation Results}

The first simulation case was run under AWGN channel condition and the results are plotted as shown in Fig. 2. For fixed $E_{b} / N_{o}$ at $0 \mathrm{~dB}$, the number of bits per symbol is varied between 1 and 10 . The results show that, the best BER performance of this model for RS $(7,3)$ code is achieved without code interleaving but with punctured code. Even though it starts with the worst BER performance, 0.6111, it gets settled with the best performance, 0.1667 for higher values of number of bits per symbol. There is an improvement of around $50 \%$ in BER performance with increased number of bits per symbol.

Figure 3 shows the BER performance of the second simulation case which was run by varying $E_{b} / N_{o}$ in the interval $(-8,8)$ with a step size $2 \mathrm{~dB}$. As shown, this proposed simulink model gives its best BER performance, 0.1667 and the performance is almost same irrespective of whether the RS code is punctured or not. This simulation case has a performance variation of around $30 \%$. 




Fig. 2. Bit Error Rate under AWGN channel for $E_{b} / N_{o}=0 d B$

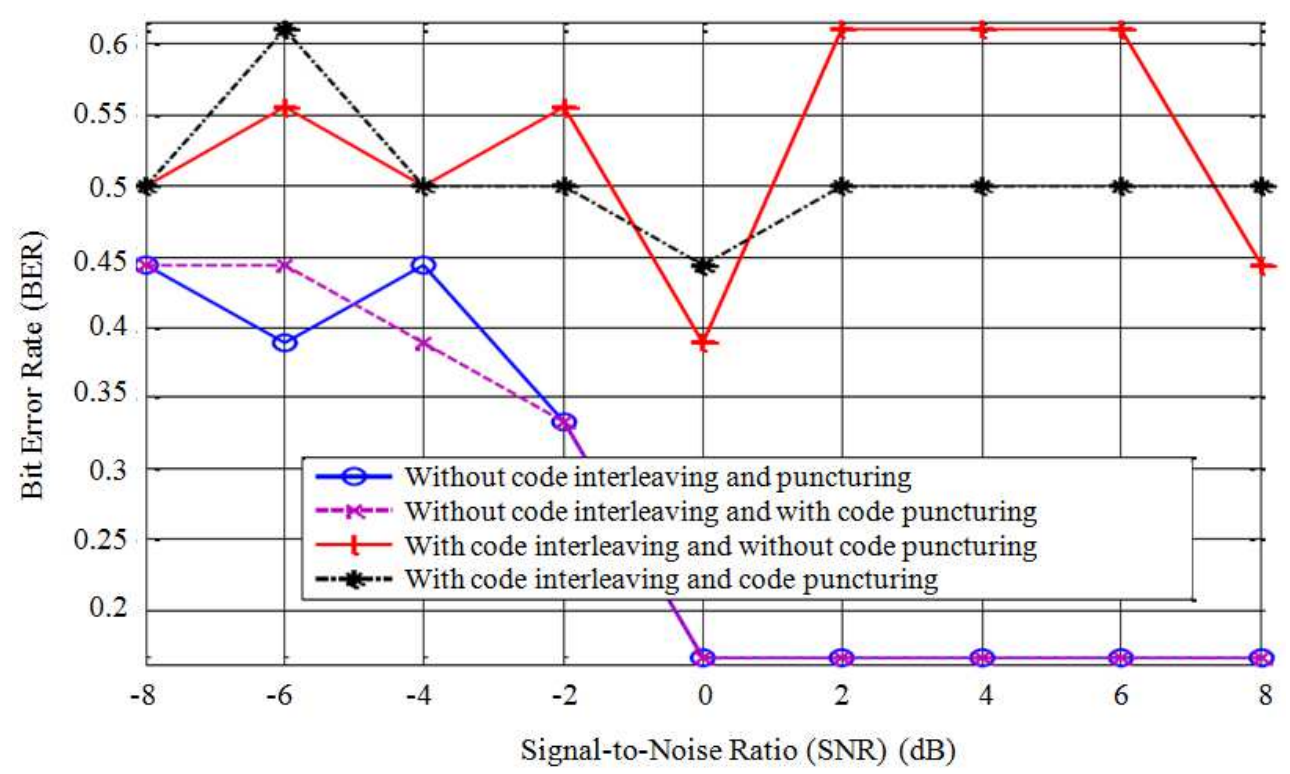

Fig. 3. Bit Error Rate under AWGN channel for Number of bits per symbol =9

The simulation results for the third case plotted in Fig. 4 are BER performance of this model under multipath Rayleigh fading channel with average path gain vector fixed at $\left[\begin{array}{ll}0 & 0\end{array}\right] \mathrm{dB}$ and various values of average path delay vector as listed in the Table 1 . This simulation result shows that, the proposed simulink model provides its best BER performance towards increased value of average path delay vector (s). Its performance increases from 0.4444 to 0.1667 i.e., an improvement of around $30 \%$ in its BER performance.

This appreciable performance improvement is achieved without code interleaving irrespective of whether the code is punctured or not. However, there is a slight performance enhancement with punctured RS code.

The fourth simulation, shown in Fig. 5 is also run under Rayleigh fading channel but with fixed average path delay vector $=\left[\begin{array}{ll}0 & 5 \mathrm{e}^{-3}\end{array}\right] \mathrm{s}$ and varied average path gain vector as listed in Table 2 . These simulation results shows that, the proposed model provides its best BER performance, 0.1667 for earlier value of average path gain vector but the performance goes worse as the value move towards positive quantities. But still the performance variation is about $16 \%$ only. 


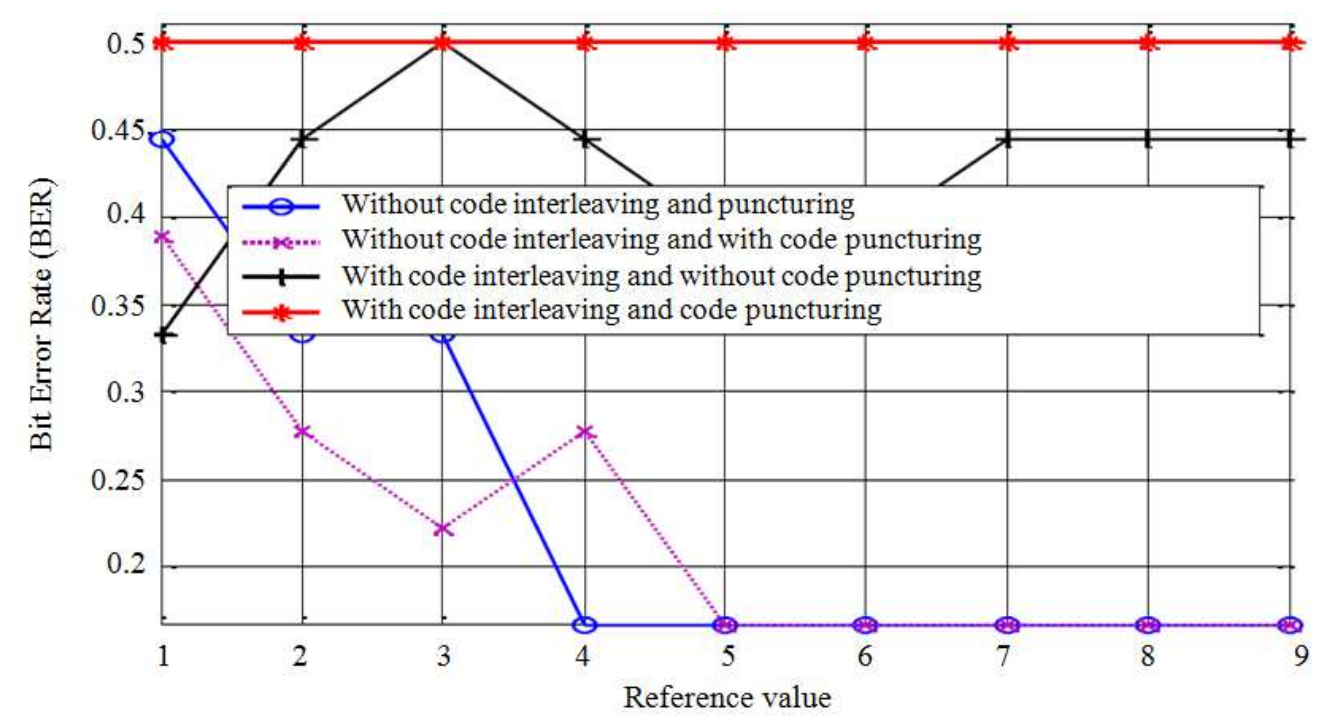

Fig. 4. Bit Error Rate under Rayleigh fading channel for Average path gain vector $=\left[\begin{array}{ll}0 & 0\end{array}\right] \mathrm{dB}$

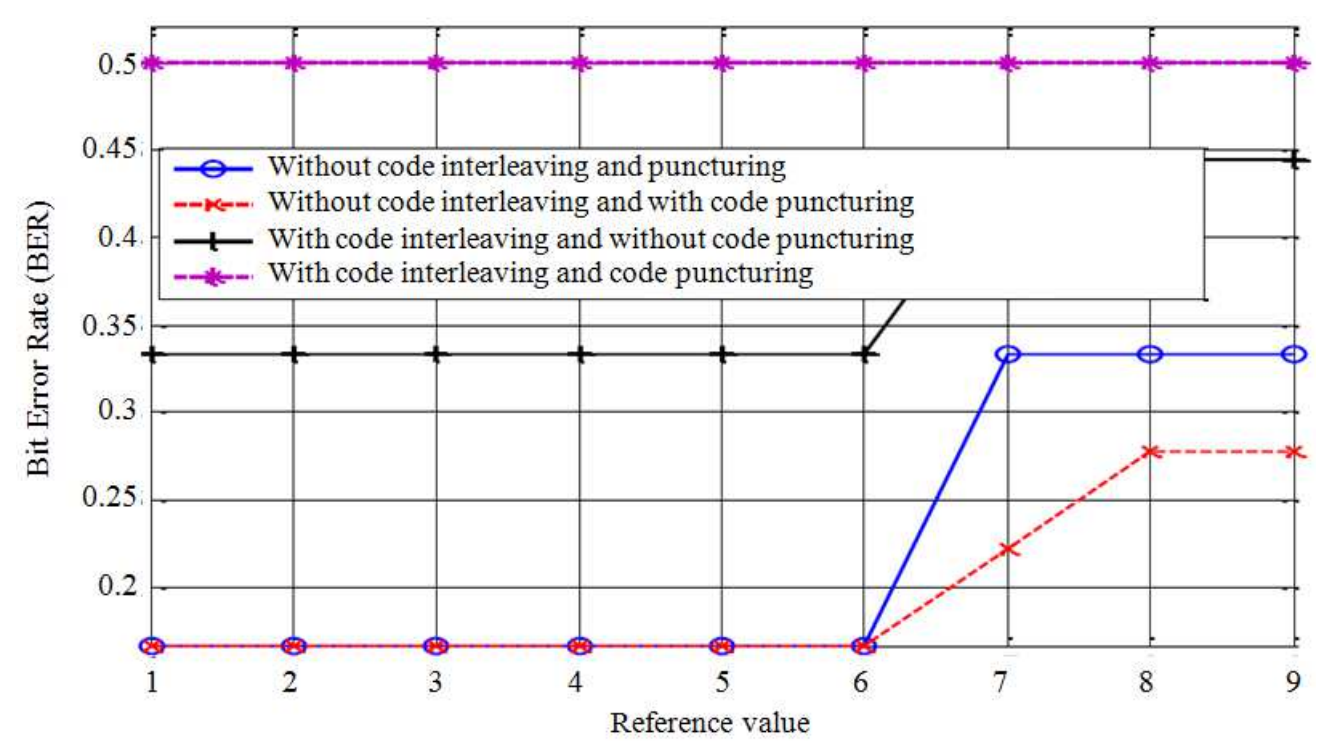

Fig. 5. Bit Error Rate under Rayleigh fading channel for Average path delay vector $=\left[\begin{array}{ll}0 & 5 \mathrm{e}^{-3}\end{array}\right] \mathrm{s}$

Table 1. X-axis reference value vs. Actual value for average path delay vector with reference to Fig. 4

\begin{tabular}{|c|c|}
\hline $\begin{array}{l}\text { Reference value } \\
\text { mentioned in X-axis }\end{array}$ & $\begin{array}{l}\text { Corresponding actual value of } \\
\text { Average path delay vector (s) }\end{array}$ \\
\hline 1 & {$\left[\begin{array}{lll}0 & 1 \mathrm{e}^{-3}\end{array}\right]$} \\
\hline 2 & {$\left[\begin{array}{lll}0 & 2 \mathrm{e}^{-3}\end{array}\right]$} \\
\hline 3 & {$\left[\begin{array}{lll}0 & 3 \mathrm{e}^{-3}\end{array}\right]$} \\
\hline 4 & {$\left[\begin{array}{lll}0 & 4 \mathrm{e}^{-3}\end{array}\right]$} \\
\hline 5 & {$\left[\begin{array}{lll}0 & 5 \mathrm{e}^{-3}\end{array}\right]$} \\
\hline 6 & {$\left[\begin{array}{lll}0 & 6 \mathrm{e}^{-3}\end{array}\right]$} \\
\hline 7 & {$\left[\begin{array}{lll}0 & 7 \mathrm{e}^{-3}\end{array}\right]$} \\
\hline 8 & {$\left[\begin{array}{lll}0 & 8 \mathrm{e}^{-3}\end{array}\right]$} \\
\hline 9 & {$\left[09 \mathrm{e}^{-3}\right]$} \\
\hline
\end{tabular}

Table 2. X-axis reference value vs. Actual value for average path delay vector with reference to Fig. 5

\begin{tabular}{|c|c|}
\hline $\begin{array}{l}\text { Reference value } \\
\text { mentioned in } \mathrm{X} \text {-axis }\end{array}$ & $\begin{array}{l}\text { Corresponding actual value of } \\
\text { Average path gain vector }(\mathrm{dB})\end{array}$ \\
\hline 0 & {$[0-8]$} \\
\hline 1 & [0-6] \\
\hline 2 & [0-4] \\
\hline 3 & {$[0-2]$} \\
\hline 4 & {$\left[\begin{array}{ll}0 & 0\end{array}\right]$} \\
\hline 5 & {$\left[\begin{array}{ll}0 & 2\end{array}\right]$} \\
\hline 6 & [0 4] \\
\hline 7 & {$\left[\begin{array}{ll}0 & 6\end{array}\right]$} \\
\hline 8 & {$\left[\begin{array}{ll}0 & 8\end{array}\right]$} \\
\hline
\end{tabular}


Again appreciable BER performance is achieved without code interleaving and code puncturing, moreover there is no much performance variation for whether the code is punctured or not.

The fifth simulation shown in Fig. 6 is run under multipath Rician fading channel for fixed average path gain vector $\left[\begin{array}{ll}0 & 0\end{array}\right] \mathrm{dB}$ with average path delay vector varied over a specified range. As shown above, this proposed simulink model gives its best BER performance, 0.2778 without code interleaving but with punctured code and this simulation has a performance variation approximately more than $50 \%$, especially its performance is poor when the code is interleaved without any regard to puncturing the codes.

The sixth simulation, shown in Fig.7 is also run under multipath Rician fading channel for fixed average path delay vector $=\left[\begin{array}{ll}0 & 5 \mathrm{e}^{-3}\end{array}\right] \mathrm{s}$ with average path gain vector varied over a specified range.

The simulation result shows that, under this condition, the best BER performance, 0.1667 is achieved without interleaving the code irrespective of whether the code is punctured or not i.e., the performance is almost similar for both the cases. This simulation has its performance variation of about $60 \%$ and code interleaving does not yield appreciable BER performance.

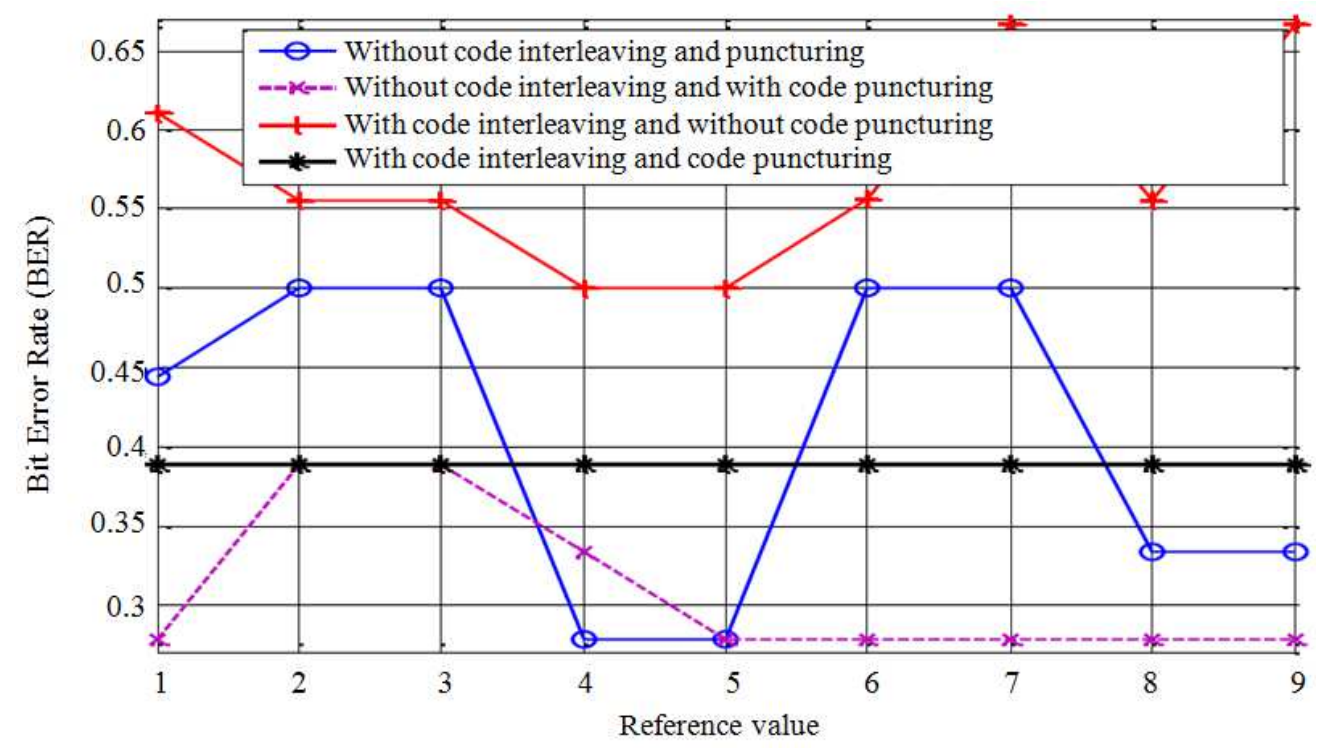

Fig. 6. Bit Error Rate under Rician fading channel for Average path gain vector $=\left[\begin{array}{ll}0 & 0\end{array}\right] \mathrm{dB}$

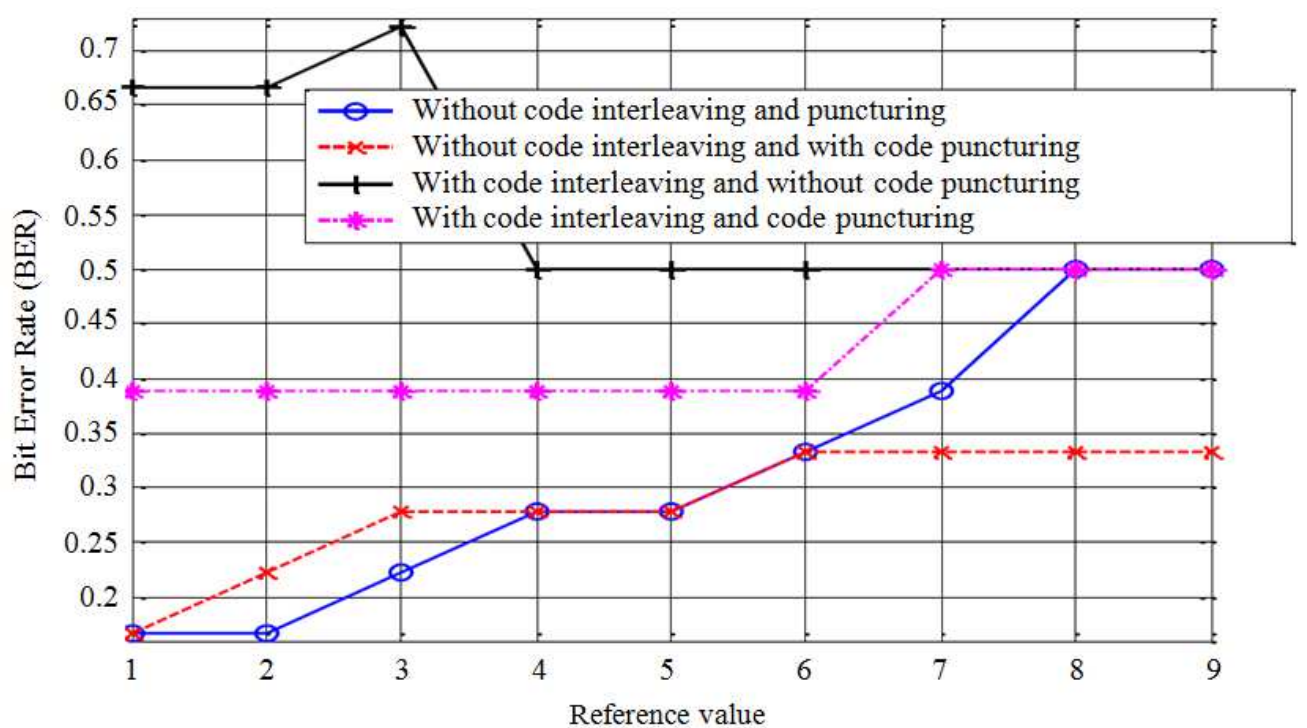

Fig. 7. Bit Error Rate under Rician fading channel for Average path delay vector $=\left[\begin{array}{ll}0 & 5 \mathrm{e}^{-3}\end{array}\right] \mathrm{s}$ 


\section{Conclusion}

Even though interleaved code words are comparatively secured, it is not suitable for a simulation environment discussed in this paper. Besides its poor BER performance, implementation of code interleaverdeinterleaver pair requires complex hardware configuration which increases power consumed by the transceiver circuitry. So, code interleaving may be a right choice for the wireless scenario with uninterrupted power supply. Puncturing the code reduces the overhead of transmitter but however it increases that of receiver. So, puncturing of code should have appreciable trade-off between BER performance and power consumption. However, the difference is much less.

\section{Acknowledgment}

The authors would like to thank the editor and anonymous referees for their valuable comments to improve the quality of this paper.

\section{Funding Information}

The authors have no support or funding to report.

\section{Author's Contributions}

To our best knowledge, this work is the first attempt to analyse BER performance of RS coded FSK scheme over AWGN, Rayleigh and Rician fading channels with RS code interleaving and puncturing. In an attempt to propose and analyze the performance of energy efficient communication scheme for low power wireless sensor nodes, authors utilize the functionalities described for MATLAB/Simulink after the literature review.

\section{Ethics}

The author's hereby declare that, this work is their own contribution and hence there would not be any ethical issues related to this manuscript.

\section{References}

El-Bendary, M.A.M., H. Kasban and M.A.R. El-Tokhy, 2014. Interleaved Reed-Solomon codes with code rate switching over wireless communications channels. Proceedings of the 2nd International Conference on Advanced in Computer, Jul. 8-9, Paris, France, 1-9.
Khan, M.K., K. Mulvaney, P. Quinlan, S. O’Mahony and C. Billon et al., 2011. On the use of ReedSolomon codes to extend link margin and communication range in low-power wireless networks. ISSC, Trinity College Dublin.

Korrapati, V., M.V.D. Prasad, D.V. Reddy and G.A. Tej, 2013. A study on performance evaluation of Reed Solomon codes through an AWGN channel model for an efficient communication system. Int. J. Eng. Trends Technol., 4: 1038-1041.

Kumar, S. and R. Gupta, 2011. Bit error rate analysis of reed-Solomon code for efficient communication system. Int. J. Comput. Applic., 30: 11-14. DOI: $10.5120 / 3710-5174$

Mahajan, S. and G. Singh, 2011. BER Performance of Reed-Solomon Code Using M-ary FSK Modulation in AWGN Channel. Int. J. Adv. Sci. Technol., 3: 7-15.

Nguyen, P., H. Vinh, T. Quang and T. Miyoshi, 2012. Multi-hop Reed-Solomon encoding scheme for image transmission on wireless sensor networks. Proceedings of the 4th International IEEE Conference on Communications and Electronics, Aug. 1-3, IEEE Xplore Press, Hue, pp: 74-79. DOI: 10.1109/CCE.2012.6315874

Shrivastava, P. and U.P. Singh, 2013. Error detection and correction using Reed Solomon codes. Int. J. Adv. Res. Comput. Sci. Software Eng., 3: 965-969.

Shrivastava, P. and U.P. Singh, 2013. Error detection and correction using Reed Solomon codes. Int. J. Adv. Res. Comput. Sci. Software Eng., 3: 965-969.

Vibha, R. and R. Nag, 2013. Performance study on the suitability of Reed-Solomon codes in communication systems. CT Int. J. Inform. Commun. Technol., 1: 13-15.

Wang, H., L. Xing and H.E. Michel, 2010. ReedSolomon code based green and survivable communications using selective encryption. Int. J. Performab. Eng., 6: 297-299. 